\title{
DIMENSIONS OF MULTIPLE DEPRIVATION IN ROMA POPULATION - A DESCRIPTION OF THE ROMA ACCORDING TO THE HOUSEHOLD BUDGET SURVEY 2016
}

\author{
Bianca BULIGESCU 1 \\ DOI: $10.35782 / J C P P .2019 .1 .03$
}

\begin{abstract}
The current article compares Roma with Romanian population in terms of various dimensions using data from the Household Budget Survey 2016. The current article aims to illustrate the multiple deprivations of the Roma population. The article is descriptive and aims to capture a snapshot of the Roma population using a large dataset. The article finds the traditional structure of the Roma family is preserved with Roma women more likely to be housewives, men more likely to be self employed workers, Roma have a lower educational status and are more likely to be poor.
\end{abstract}

Keywords: Roma, deprivations, inequalities, poverty, Household Budget Survey 2016

\section{Introduction}

This article compares Roma with the Romanian population in terms of various dimensions using data from the Household Budget Survey 2016. The used method is descriptive analysis by doing simple cross-tabulations.

According to the latest report on Roma inclusion index published by Decade for Roma Inclusion 2005-2015 Secretariat Foundation (2015, p.56) despite an improvement in the situation of Roma in Romania, three-fourths of them live at risk of poverty, and about $40 \%$ in absolute poverty ${ }^{2}$. Roma live on $60 \%$ less income than the total population, while one-third of them perceive themselves as being discriminated against. Zamfir C. describes in a synthetic manner most of the issues the Roma face: "they have a poor socioeconomic situation, characterized by severe poverty, a marginal position: low education, marginal fluctuating employment, poor living conditions, accentuated by a traditional attitude of discrimination,

1 Researcher, Research Institute for Quality of Life, Romanian Academy; UNU-MERIT, Maastricht University. Email: bianca.buligescu@iccv.ro

2 Data comes from various sources and multiple surveys. 
negative image, and a traditional lifestyle which is characterized as a survival strategy in the position of severe and severe poverty marginalization that accentuates separation from the rest of the population." (Zamfir C. in Zamfir E. (eds.), 2015, p.562). Roma that live in Roma communities face multiple deprivations and in fact they live in pockets of multidimensional poverty characterized by: "low levels of employment, severe poverty, precarious housing conditions, low level of participation in education, marginal access to bealth care, minimal access to public utilities (water, sewage, electricity), spatial isolation with low level of access to transportation" (Zamfir C. in Zamfir E. (eds.), 2015, p.562). The majority of Roma live in compact communities at the peripheries of settlements (Zamfir C. in Zamfir E. (eds.), 2015, p.563). Spatial segregation is enhanced by a number of policy related factors: "returning the houses to their former owners, the increase in the prices of properties, the decrease in the level of salaries, precarious employment with low and insecure earnings, the neoliberal policy of a small state" (Zamfir C. in Zamfir E. (eds.), 2015, p.563). There is an intergenerational transmission of poverty that remains constant throughout time (Zamfir C. in Zamfir E. (eds.), 2015, p.563). "The majority of Roma reproduces from one generation to the next a low socioeconomic marginal status, being enclosed in a traditional lifestyle which is adapted to a poverty and marginal position" (Zamfir C. in Zamfir E. (eds.), 2015, p.566).

Critical data from the official statistics office related to the social inclusion of Roma and their families are often missing, making it difficult to monitor the implementation of the EU Charter of Fundamental Rights of the European Union. Data is available through a series of surveys but the surveys have different methodologies making it difficult to monitor the situation of Roma. Data on Roma children and their families are often missing due to several factors:

- First, the Roma children represent a minority which is a vulnerable group (at high risk of poverty) being hard to reach and often living in the outskirts of town/cities or in excluded areas spread over a large territory (some live in compact Roma communities whereas others live in spatially dispersed areas)

- Second, they are often discriminated against and tend to under-report their ethnicity in official statistics such as the Census data ${ }^{1}$ or they are integrated in the mainstream population and make themselves invisible for fear of negative associations others may have on their ethnic group

- Third, not all Roma people speak the Romani language

- Fourth, declaring ethnic affiliation is a personal option due to the implementation of Directive 95/46/EC transposed in national legislation Law no. $677 / 2001$ on the protection of individuals with regard to the processing of personal data and free movement ${ }^{2}$.

\footnotetext{
1 To illustrate these issues, 2011 Census data under-reports the Roma population estimating it at $3.08 \%$ of the population whereas data from the Council of Europe estimate it at $8.63 \%$ of the population. (Council of Europe, 2012: 20)

2 The collection of data in regard to ethnicity is prohibited with exceptions, such as: a) when the data subject has expressly given his/her consent for such data processing; b) when the
} 
- Fifth, many governments collect data about the Roma from their national statistics sources such as Census data and there is a huge discrepancy between Census estimates who tend to under-estimate and NGO estimates who tend to over-estimate.

A recent report by Roma Decade (2015) collecting data from various sources lists the main gaps in indicators: "data are completely missing for special schools, long-term unemployment and homelessness. Recent data disaggregated by gender in housing, health (except access to health insurance) and cross-cutting areas are missing, and baseline data are missing on preschool education, informal employment, last and no employment experience and youth NEETS rate (youth not in school nor in employment), property documents and housing segregation, access to bealth insurance and discrimination".

Furthermore, existing data have been collected on a project basis (See Annex 1), surveying the vulnerable groups using a one-time survey design making it difficult to monitor progress on the situation of Roma children and their families.

\section{Methodology}

The current methodology builds upon the Household Budget Survey 2016 (HBS) which collects information about households' consumption patterns incomes and work in Romania. The total sample size is 60569 , the sample size or Romanian population is 54775 and the sample size of Roma population is 1381 of individuals. The sample size for Roma population illustrates that Roma are over-represented in rural areas. Data from HBS has been previously used in a report by the World Bank on "Achieving Roma inclusion in Romania, What does it take?" for the year 2011. The main research question what are the main patterns of inequality between the Roma and Romanians. The analysis will be further developed across other variables in the HBS data such as: level of educational status, occupational status, civil status, the reason of absence from work, professional status, type of contract, if they receive free goods or services from work. The analysis will shed some light on the income poverty status of Roma and whether they are more prone to social assistance than native Romanians.

Based on the Charter of Human Rights we identified the following dimensions of deprivations: health, nutrition, education, living conditions, income/expenditure, employment, cross-cutting indicators, the potential list of indicators can be found in the table below. For some indicators it might be the case that we cannot measure them with the data at hand.

processing is carried out as part of the legitimate activities of a foundation, association, or of any other nonprofit organization with a political, philosophical, religious or trade union profile, provided that the data subject is a member of that organization or has regular contacts with the organization in its activity profile, and provided that the data shall not be disclosed to a third party without the data subject's consent; and c) when the processing refers to data made expressly public in a clear way by the data subject. This increases the difficulties to have comprehensive statistics. However, it is our task to overcome such barriers, and make available data to serve the interest of the project. 
Table no 1. Dimensions of deprivation.

\begin{tabular}{|l|l|l|}
\hline $\begin{array}{c}\text { Dimensions of Human } \\
\text { Rights and Human } \\
\text { Development }\end{array}$ & $\begin{array}{c}\text { Dimensions } \\
\text { of deprivation }\end{array}$ & \multicolumn{1}{|c|}{ Potential List of Indicators } \\
\hline Right to health & Health & $\begin{array}{l}\text { Life Expectancy, Effective access to health } \\
\text { care services, Early pregnancy rates }\end{array}$ \\
\hline Right to health & Nutrition & $\begin{array}{l}\text { Malnutrition, Hunger, Consumption of } \\
\text { protein per week }\end{array}$ \\
\hline $\begin{array}{l}\text { Equality of opportunities in } \\
\text { education access }\end{array}$ & Education & $\begin{array}{l}\text { Educational attainment, education by age, } \\
\text { education by area }\end{array}$ \\
\hline $\begin{array}{l}\text { Right to adequate standard } \\
\text { of living adequate nutrition, } \\
\text { clothing and housing }\end{array}$ & $\begin{array}{l}\text { Living } \\
\text { conditions }\end{array}$ & $\begin{array}{l}\text { Dwelling conditions, no drinking water, no } \\
\text { electricity at home, not holding property } \\
\text { documents, segregated housing, } \\
\text { overcrowding }\end{array}$ \\
\hline $\begin{array}{l}\text { Right to adequate standard } \\
\text { of living }\end{array}$ & $\begin{array}{l}\text { Income/Expe } \\
\text { nditure }\end{array}$ & $\begin{array}{l}\text { Income sources, Expenditure structure, } \\
\text { percentage of Roma children by income } \\
\text { quantiles, social assistance benefits }\end{array}$ \\
\hline $\begin{array}{l}\text { Right to adequate standard } \\
\text { of living and support for } \\
\text { families, no employment or } \\
\text { exploitation of minors }\end{array}$ & Employment & $\begin{array}{l}\text { Informal work, Employment structure by } \\
\text { area, Employment structure by working } \\
\text { age, Types of contract (temporary, part- } \\
\text { time), Unemployment, child labour }\end{array}$ \\
\hline $\begin{array}{l}\text { EU Charter of Fundamental } \\
\text { Rights of the European } \\
\text { Union }\end{array}$ & $\begin{array}{l}\text { Cross-cutting } \\
\text { indicators }\end{array}$ & $\begin{array}{l}\text { At-risk of poverty, At-risk of material } \\
\text { deprivation, Absolute poverty, Average } \\
\text { income, }\end{array}$ \\
\hline
\end{tabular}

Source: Charter of Human Rights

\section{Results}

We find in 2016 data for HBS that Roma have a high birth rate and a lower life span than native Romanians (see Figure 1). The average age of Roma is 33 years old whereas the average age of Romanians is 49 , therefore Roma have a relatively young population whereas Romanians started to have an ageing problem of the population. Further we can see that Romanians have an equal distribution between urban and rural areas whereas Roma are more predominant in rural areas.

When we look at the civil status, we find that among Roma, 36\% are married, about $12 \%$ live in partnership and $42 \%$ are single, reflecting the fact that Roma have younger generations and tend towards cohabitation. Among the Romanians, 53\% are married, only $3 \%$ live in a partnership relationship, $24 \%$ live alone and $15 \%$ are widows, compared to only $6 \%$ of Roma, reflecting a longer life expectancy of Romanians. 
Figure 1. Age distribution by nationality

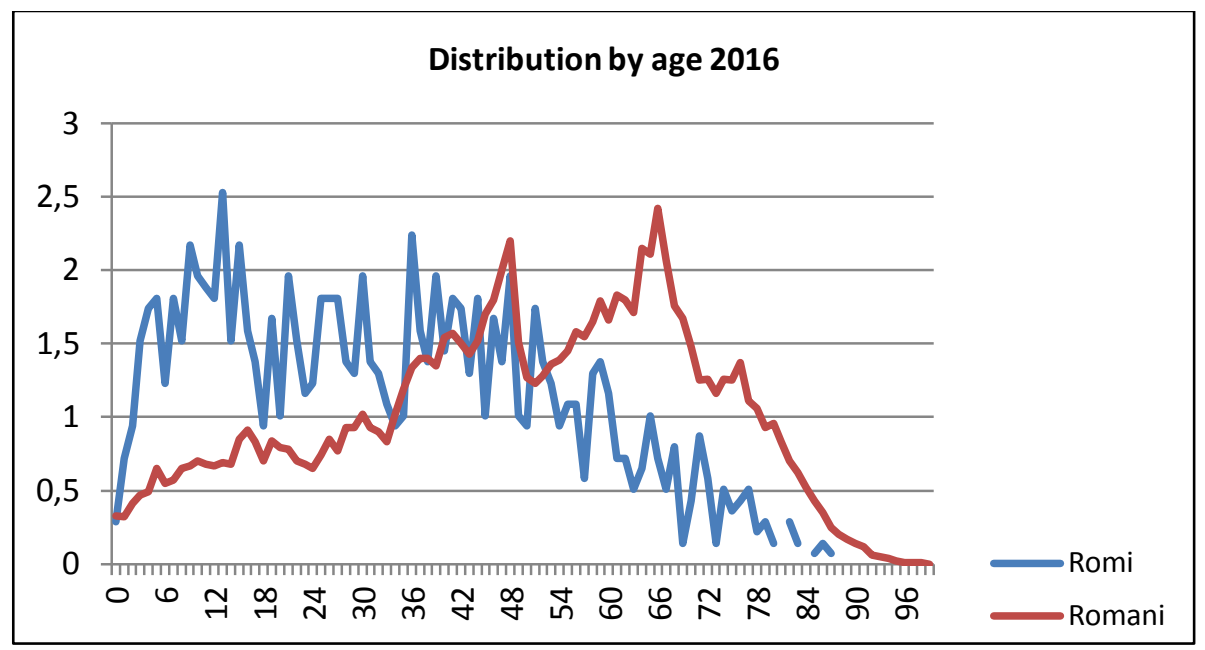

Source: Household Budget Survey, 2016

Notes: In blue Roma, in red Romanians

Table 2. Age summary statistic by nationality

\begin{tabular}{|l|r|l|l|r|r|}
\hline \multicolumn{1}{|c|}{ Age } & N sample & \multicolumn{1}{c|}{ Average } & Standard Deviation & \multicolumn{1}{c|}{ Min } & \multicolumn{1}{c|}{ Max } \\
\hline Roma & 1381 & 33.46 & 20.47 & 0 & 87 \\
\hline Romanian & 54775 & 48.76 & 21.50 & 0 & 100 \\
\hline
\end{tabular}

Source: Household Budget Survey, 2016

Table 3. Distribution by area and nationality

\begin{tabular}{|l|r|r|r|r|r|r|}
\hline Area & \multicolumn{1}{|l|}{ Romanian } & \multicolumn{1}{l|}{ Hungarian } & \multicolumn{1}{l|}{ Roma } & \multicolumn{1}{l|}{ Germans } & \multicolumn{1}{l|}{ Other } & \multicolumn{1}{c|}{ Total } \\
\hline \hline \multirow{3}{*}{ Urban } & 26,960 & 1,614 & 513 & 33 & 116 & 29,236 \\
\cline { 2 - 8 } & 49.22 & 39.26 & 37.15 & 51.56 & 48.74 & 48.27 \\
\hline \multirow{3}{*}{ Rural } & 27,815 & 2,497 & 868 & 31 & 122 & 31,333 \\
\hline \multirow{3}{*}{ Total } & 50.78 & 60.74 & 62.85 & 48.44 & 51.26 & 51.73 \\
\cline { 2 - 7 } & 54,775 & 4,111 & 1,381 & 64 & 238 & 60,569 \\
\hline
\end{tabular}

Source: Household Budget Survey, 2016

Table 4. Civil status by nationality

\begin{tabular}{|l|r|r|r|r|r|r|}
\hline Civil status & \multicolumn{1}{|c|}{ Romanian } & \multicolumn{1}{c|}{ Hungarian } & \multicolumn{1}{c|}{ Roma } & \multicolumn{1}{c|}{ Germans } & Others & \multicolumn{1}{c|}{ Total } \\
\hline \multirow{3}{*}{ Married } & 29,242 & 2,116 & 501 & 33 & 134 & 32,026 \\
\hline \multirow{3}{*}{ Partnership } & 53.39 & 51.47 & 36.28 & 51.56 & 56.3 & 52.88 \\
\cline { 2 - 7 } & 1,556 & 167 & 160 & 2 & 9 & 1,894 \\
\hline
\end{tabular}




\begin{tabular}{|c|c|c|c|c|c|c|}
\hline Civil status & Romanian & Hungarian & Roma & Germans & Others & Total \\
\hline \multirow[b]{2}{*}{ Divorced } & 2,688 & 163 & 33 & 5 & 10 & 2,899 \\
\hline & 4.91 & 3.96 & 2.39 & 7.81 & 4.2 & 4.79 \\
\hline \multirow[b]{2}{*}{ Widow } & 8,283 & 657 & 86 & 14 & 40 & 9,080 \\
\hline & 15.12 & 15.98 & 6.23 & 21.88 & 16.81 & 14.99 \\
\hline \multirow[b]{2}{*}{ Single } & 12,641 & 987 & 581 & 9 & 44 & 14,262 \\
\hline & 23.08 & 24.01 & 42.07 & 14.06 & 18.49 & 23.55 \\
\hline \multirow[b]{2}{*}{ Separated } & 365 & 21 & 20 & 1 & 1 & 408 \\
\hline & 0.67 & 0.51 & 1.45 & 1.56 & 0.42 & 0.67 \\
\hline \multirow[b]{2}{*}{ Total } & 54,775 & 4,111 & 1,381 & 64 & 238 & 60,569 \\
\hline & 100 & 100 & 100 & 100 & 100 & 100 \\
\hline
\end{tabular}

Source: Household Budget Survey, 2016

As we can see from the Table below, the data at hand the Household Budget Survey contains information about 33\% household heads, $20 \%$ partners, $36 \%$ are daughters/sons, $2 \%$ are dauthers in law/sons in law, $6 \%$ are nephews/nieces and about $1.4 \%$ are without any blood relation.

Table 5. Roma household structure

\begin{tabular}{|l|r|r|r|}
\hline \multicolumn{1}{|c|}{ Roma Household structure } & \multicolumn{1}{c|}{ Freq. } & \multicolumn{1}{c|}{ Percent } & \multicolumn{1}{c|}{ Cum. } \\
\hline Hosehold head & 452 & 32.73 & 32.73 \\
\hline Husband/wife/partener & 287 & 20.78 & 53.51 \\
\hline Daughter/son & 491 & 35.55 & 89.07 \\
\hline Daughter in law/son in law & 28 & 2.03 & 91.09 \\
\hline Nephew/nice & 78 & 5.65 & 96.74 \\
\hline Dad/mother/Mother in law/ Dad in law & 5 & 0.36 & 97.1 \\
\hline Brother/sister/ brother in law/ sister in law & 9 & 0.65 & 97.76 \\
\hline Other relatives & 10 & 0.72 & 98.48 \\
\hline Children in foster care & 1 & 0.07 & 98.55 \\
\hline Without any blood relation & 20 & 1.45 & 100 \\
\hline Total & 1,381 & 100 & \\
\hline
\end{tabular}

Source: Household Budget Survey, 2016

When we look at educational level, we find that among Roma 24-61 years aged, about $8 \%$ of the Roma are without any school completed, compared to only $0.26 \%$ for Romanians, 36\% have completed primary classes compared to 2\% for Romanians, $45 \%$ of Roma have completed gymnasium, compared with 14\% for Romanians, 5\% have completed a vocational school compared to $24 \%$ for Romanians, only $2 \%$ of Roma have completed a high school compared to $31 \%$ of Romanians, about $16 \%$ of Romanians have higher education, according to ABF, compared with no 1\% of Roma. From the data available, it is clear that there are educational deficiencies among Roma. If we further analyze the distribution of the educational level by age and look at it over the generations, we notice that about $7 \%$ of the population aged between 25 and 30 have not graduated from a school, compared to about $20 \%$ for those over the age of 66 , who were educated and worked during communism, but the percentage increases with the aging. 
Approximately $35 \%$ of Roma aged between 25 and 30 have completed primary classes, compared with $62 \%$ of those over the age of 66 . Approximately $47 \%$ of Roma aged between 25 and 30 have graduated from gymnasium, compared with 14\% of over 66 . Younger generations are characterized by a higher level of school education, about 3\% of those aged between 25 and 30 graduating from high school, compared with $0 \%$ of those over 66. By comparison, about 38\% of Romanians with ages between 25 and 30 have graduated from high school, compared with $8 \%$ among those over 65 . About $28 \%$ of Romanians aged between 25 and 30 have higher education, compared with only $5 \%$ among those over 65 years.

Table 6. Educational attainment by nationality

\begin{tabular}{|c|c|c|c|c|c|c|}
\hline Educational attainment & $\begin{array}{c}\text { Roma } \\
\text { nian }\end{array}$ & $\begin{array}{c}\text { Hung } \\
\text { arian }\end{array}$ & Roma & $\begin{array}{c}\text { Germ } \\
\text { ans }\end{array}$ & $\begin{array}{c}\text { Other } \\
\mathrm{s}\end{array}$ & Total \\
\hline \multirow{2}{*}{ Without any school completed } & 2,199 & 140 & 251 & 1 & 6 & 2,597 \\
\hline & 4.01 & 3.41 & 18.18 & 1.56 & 2.52 & 4.29 \\
\hline \multirow{2}{*}{ Kindengartnen } & 1,252 & 99 & 76 & 1 & 3 & 1,431 \\
\hline & 2.29 & 2.41 & 5.5 & 1.56 & 1.26 & 2.36 \\
\hline \multirow{2}{*}{ Primary school (classes $0-4$ ) } & 5,759 & 338 & 477 & 6 & 30 & 6,610 \\
\hline & 10.51 & 8.22 & 34.54 & 9.38 & 12.61 & 10.91 \\
\hline \multirow{2}{*}{ Gymnasium (classes 5-8) } & 10,890 & 1,024 & 460 & 17 & 54 & 12,445 \\
\hline & 19.88 & 24.91 & 33.31 & 26.56 & 22.69 & 20.55 \\
\hline \multirow{2}{*}{ Vocational school } & 10,993 & 819 & 46 & 11 & 49 & 11,918 \\
\hline & 20.07 & 19.92 & 3.33 & 17.19 & 20.59 & 19.68 \\
\hline \multirow{2}{*}{ High-school (class 9th or 10) } & 3,380 & 355 & 40 & 1 & 10 & 3,786 \\
\hline & 6.17 & 8.64 & 2.9 & 1.56 & 4.2 & 6.25 \\
\hline \multirow{2}{*}{ Highschool (class 11 th or 12 th $/ 13$ th) } & 12,231 & 877 & 25 & 17 & 60 & 13,210 \\
\hline & 22.33 & 21.33 & 1.81 & 26.56 & 25.21 & 21.81 \\
\hline \multirow{2}{*}{$\begin{array}{l}\text { Vocational school after } \\
\text { highschool/tehnic speciality }\end{array}$} & 2,609 & 196 & 3 & 3 & 1 & 2,812 \\
\hline & 4.76 & 4.77 & 0.22 & 4.69 & 0.42 & 4.64 \\
\hline \multirow{2}{*}{ University I (BA studies) } & 566 & 45 & 0 & 0 & 4 & 615 \\
\hline & 1.03 & 1.09 & 0 & 0 & 1.68 & 1.02 \\
\hline \multirow{2}{*}{ University II (MA studies) } & 172 & 16 & 0 & 1 & 3 & 192 \\
\hline & 0.31 & 0.39 & 0 & 1.56 & 1.26 & 0.32 \\
\hline \multirow{2}{*}{ University III (PhD) } & 92 & 10 & 1 & 0 & 0 & 103 \\
\hline & 0.17 & 0.24 & 0.07 & 0 & 0 & 0.17 \\
\hline \multirow{2}{*}{ University long duration (4-6 years) } & 4,503 & 188 & 2 & 6 & 18 & 4,717 \\
\hline & 8.22 & 4.57 & 0.14 & 9.38 & 7.56 & 7.79 \\
\hline \multirow{2}{*}{ Postuniversity } & 111 & 4 & 0 & 0 & 0 & 115 \\
\hline & 0.2 & 0.1 & 0 & 0 & 0 & 0.19 \\
\hline \multirow{2}{*}{$\mathrm{PhD} /$ postdoctoral studies } & 18 & $\overline{0}$ & $\overline{0}$ & 0 & 0 & 18 \\
\hline & 0.03 & 0 & 0 & 0 & 0 & 0.03 \\
\hline \multirow{2}{*}{ Total } & 54,775 & 4,111 & 1,381 & 64 & 238 & 60,569 \\
\hline & 100 & 100 & 100 & 100 & 100 & 100 \\
\hline
\end{tabular}

Source: Household Budget Survey, 2016 
Table 7. Level of educational attainment for 15-61 years aged by nationality

\begin{tabular}{|c|c|c|c|c|c|c|}
\hline $\begin{array}{l}\text { Level of educational attainment for } \\
\text { the working age 15-61 }\end{array}$ & $\begin{array}{l}\text { Roma- } \\
\text { nian }\end{array}$ & $\begin{array}{l}\text { Hunga- } \\
\text { rian }\end{array}$ & Roma & Germans & Others & Total \\
\hline \multirow[b]{2}{*}{ Without any school } & 86 & 6 & 64 & 0 & 0 & 156 \\
\hline & 0.27 & 0.25 & 6.99 & 0 & 0 & 0.44 \\
\hline \multirow[b]{2}{*}{ Kindengartnen } & 10 & 5 & 13 & 0 & 0 & 28 \\
\hline & 0.03 & 0.21 & 1.42 & 0 & 0 & 0.08 \\
\hline \multirow[b]{2}{*}{ Primary school (classes $0-4)$} & 645 & 48 & 305 & 1 & 7 & 1,006 \\
\hline & 2.02 & 2.03 & 33.33 & 4.35 & 4.67 & 2.85 \\
\hline \multirow[b]{2}{*}{ Gymnasium (classes 5-8) } & 5,382 & 438 & 422 & 3 & 26 & 6,271 \\
\hline & 16.9 & 18.54 & 46.12 & 13.04 & 17.33 & 17.76 \\
\hline \multirow[b]{2}{*}{ Vocational school } & 6,731 & 521 & 42 & 3 & 30 & 7,327 \\
\hline & 21.13 & 22.05 & 4.59 & 13.04 & 20 & 20.75 \\
\hline \multirow[b]{2}{*}{ High-school (class 9th or 10) } & 2,861 & 293 & 40 & 1 & 9 & 3,204 \\
\hline & 8.98 & 12.4 & 4.37 & 4.35 & 6 & 9.08 \\
\hline \multirow[b]{2}{*}{ Highschool (class 11th or 12th / 13th) } & 10,104 & 718 & 24 & 9 & 55 & 10,910 \\
\hline & 31.72 & 30.39 & 2.62 & 39.13 & 36.67 & 30.9 \\
\hline \multirow{2}{*}{$\begin{array}{l}\text { Vocational school after } \\
\text { highschool/tehnic speciality }\end{array}$} & 1,595 & 121 & 2 & 2 & 1 & 1,721 \\
\hline & 5.01 & 5.12 & 0.22 & 8.7 & 0.67 & 4.87 \\
\hline \multirow[b]{2}{*}{ University I (BA studies) } & 564 & 45 & 0 & 0 & 4 & 613 \\
\hline & 1.77 & 1.9 & 0 & 0 & 2.67 & 1.74 \\
\hline \multirow[b]{2}{*}{ University II (MA studies) } & 167 & 16 & 0 & 1 & 3 & 187 \\
\hline & 0.52 & 0.68 & 0 & 4.35 & 2 & 0.53 \\
\hline \multirow[b]{2}{*}{ University III (PhD) } & 81 & 9 & 1 & 0 & 0 & 91 \\
\hline & 0.25 & 0.38 & 0.11 & 0 & 0 & 0.26 \\
\hline \multirow[b]{2}{*}{ University long duration (4-6 years) } & 3,518 & 139 & 2 & 3 & 15 & 3,677 \\
\hline & 11.04 & 5.88 & 0.22 & 13.04 & 10 & 10.42 \\
\hline \multirow[b]{2}{*}{ Postuniversity } & 102 & 4 & 0 & 0 & 0 & 106 \\
\hline & 0.32 & 0.17 & 0 & 0 & 0 & 0.3 \\
\hline \multirow[b]{2}{*}{$\mathrm{PhD} /$ postdoctoral studies } & 7 & 0 & 0 & 0 & 0 & 7 \\
\hline & 0.02 & 0 & 0 & 0 & 0 & 0.02 \\
\hline \multirow{2}{*}{ 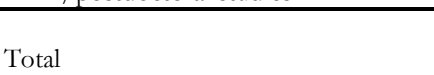 } & 31,853 & 2,363 & 915 & 23 & 150 & 35,304 \\
\hline & 100 & 100 & 100 & 100 & 100 & 100 \\
\hline
\end{tabular}

Source: Household Budget Survey, 2016

Table 8. Level of educational attainment for the working age 24-61 years by nationality

\begin{tabular}{|c|c|c|c|c|c|c|}
\hline $\begin{array}{l}\text { Level of educational } \\
\text { attainment for the } \\
\text { working age } 24-61 \\
\text { years }\end{array}$ & Romanian & Hungarian & Roma & Germans & Others & Total \\
\hline \multirow[b]{2}{*}{ Without any school } & 74 & 5 & 57 & 0 & 0 & 136 \\
\hline & 0.26 & 0.25 & 7.81 & 0 & 0 & 0.44 \\
\hline \multirow[b]{2}{*}{ Kindengartnen } & 10 & 5 & 6 & 0 & 0 & 21 \\
\hline & 0.04 & 0.25 & 0.82 & 0 & 0 & 0.07 \\
\hline \multirow{2}{*}{$\begin{array}{l}\text { Primary school (classes } \\
0-4 \text { ) }\end{array}$} & 558 & 39 & 260 & 1 & 7 & 865 \\
\hline & 1.99 & 1.92 & 35.62 & 4.55 & 5.11 & 2.8 \\
\hline \multirow[b]{2}{*}{ Gymnasium (classes 5-8) } & 3,934 & 313 & 326 & 2 & 20 & 4,595 \\
\hline & 14.06 & 15.4 & 44.66 & 9.09 & 14.6 & 14.87 \\
\hline \multirow[b]{2}{*}{ Vocational school } & 6,584 & 512 & 38 & 3 & 30 & 7,167 \\
\hline & 23.54 & 25.18 & 5.21 & 13.64 & 21.9 & 23.2 \\
\hline
\end{tabular}




\begin{tabular}{|c|c|c|c|c|c|c|}
\hline $\begin{array}{l}\text { Level of educational } \\
\text { attainment for the } \\
\text { working age } 24-61 \\
\text { years }\end{array}$ & Romanian & Hungarian & Roma & Germans & Others & Total \\
\hline \multirow{2}{*}{$\begin{array}{l}\text { High-school (class 9th } \\
\text { or } 10 \text { ) }\end{array}$} & 2,268 & 240 & 26 & 1 & 9 & 2,544 \\
\hline & 8.11 & 11.81 & 3.56 & 4.55 & 6.57 & 8.23 \\
\hline \multirow{2}{*}{$\begin{array}{l}\text { highschool (class 11th } \\
\text { or 12th / 13th) }\end{array}$} & 8,646 & 596 & 12 & 9 & 49 & 9,312 \\
\hline & 30.91 & 29.32 & 1.64 & 40.91 & 35.77 & 30.14 \\
\hline \multirow{2}{*}{$\begin{array}{l}\text { Vocational school after } \\
\text { highschool/tehnic } \\
\text { speciality }\end{array}$} & 1,566 & 116 & 2 & 2 & 1 & 1,687 \\
\hline & 5.6 & 5.71 & 0.27 & 9.09 & 0.73 & 5.46 \\
\hline \multirow{2}{*}{$\begin{array}{l}\text { University I (BA } \\
\text { studies) }\end{array}$} & 493 & 40 & 0 & 0 & 3 & 536 \\
\hline & 1.76 & 1.97 & 0 & 0 & 2.19 & 1.74 \\
\hline \multirow{2}{*}{$\begin{array}{l}\text { University II (MA } \\
\text { studies) }\end{array}$} & 164 & 16 & 0 & 1 & 3 & 184 \\
\hline & 0.59 & 0.79 & 0 & 4.55 & 2.19 & 0.6 \\
\hline \multirow[b]{2}{*}{ University III (PhD) } & 81 & 9 & 1 & 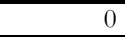 & 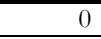 & 91 \\
\hline & 0.29 & 0.44 & 0.14 & 0 & 0 & 0.29 \\
\hline \multirow{2}{*}{$\begin{array}{l}\text { University long } \\
\text { duration (4-6 years) }\end{array}$} & 3,486 & 138 & 2 & 3 & 15 & 3,644 \\
\hline & 12.46 & 6.79 & 0.27 & 13.64 & 10.95 & 11.8 \\
\hline \multirow[b]{2}{*}{ Postuniversity } & 100 & 4 & 0 & 0 & 0 & 104 \\
\hline & 0.36 & 0.2 & 0 & 0 & 0 & 0.34 \\
\hline \multirow{2}{*}{$\begin{array}{l}\text { PhD / postdoctoral } \\
\text { studies }\end{array}$} & 7 & 0 & 0 & 0 & 0 & 7 \\
\hline & 0.03 & 0 & 0 & 0 & 0 & 0.02 \\
\hline \multirow[b]{2}{*}{ Total } & 27,971 & 2,033 & 730 & 22 & 137 & 30,893 \\
\hline & 100 & 100 & 100 & 100 & 100 & 100 \\
\hline
\end{tabular}

Source: Household Budget Survey, 2016

If we analyze the professional situation of those aged $15-61$, about $15 \%$ of the Roma are employed, compared to $51 \%$ for the Romanians. About $12 \%$ of Roma are selfemployed workers in non-agricultural fields, compared to $5 \%$ for Romanians. Approximately $17 \%$ of Roma are self-employed workers in agriculture, compared to $10 \%$ for Romanians, about $15 \%$ of Roma are unemployed according to the official definition of unemployment (unemployed and looking for a job), compared to $4 \%$ for Romanians, $4 \%$ of Roma are retired, compared to $10 \%$ in the case of Romanians, $5 \%$ are pupils, compared to only $6 \%$ for Romanians, about $23 \%$ of Roma are housewives, compared to only $8 \%$ for Romanians and about $6 \%$ represent elderly or dependent children , compared with only $2 \%$ for Romanians. From the data available, there is a traditional picture of the Roma family with women who are housewives and men who are self-employed workers rather than employees compared to the Romanian family in which both women and men work.

Table 9. Occupational status for people aged 15-61 by nationality

\begin{tabular}{|l|r|r|r|r|r|r|}
\hline $\begin{array}{c}\text { Occupational status in the } \\
\text { last 12 months }\end{array}$ & Romanian & Hungarian & Roma & Germans & Others & Total \\
\hline Forpersons aged 15-61 years & & & & & & \\
\hline \multirow{3}{*}{ Employee } & 16,368 & 1,187 & 140 & 10 & 75 & 17,780 \\
\hline \multirow{3}{*}{ Entrepreneur } & 51.39 & 50.23 & 15.3 & 43.48 & 50 & 50.36 \\
\cline { 2 - 8 } & 84 & 4 & 2 & 0 & 6 & 96 \\
\hline
\end{tabular}




\begin{tabular}{|c|c|c|c|c|c|c|}
\hline $\begin{array}{l}\text { Occupational status in the } \\
\text { last } 12 \text { months }\end{array}$ & Romanian & Hungarian & Roma & Germans & Others & Total \\
\hline \multirow{2}{*}{$\begin{array}{l}\text { Self-employed worker in non- } \\
\text { agriculture activities }\end{array}$} & 1,480 & 127 & 111 & 5 & 6 & 1,729 \\
\hline & 4.65 & 5.37 & 12.13 & 21.74 & 4 & 4.9 \\
\hline \multirow{2}{*}{$\begin{array}{l}\text { Member of a non-agriculture } \\
\text { cooperative }\end{array}$} & 11 & 8 & 3 & 0 & 0 & 22 \\
\hline & 0.03 & 0.34 & 0.33 & 0 & 0 & 0.06 \\
\hline \multirow{2}{*}{$\begin{array}{l}\text { Self-employed worker in } \\
\text { agriculture }\end{array}$} & 3,116 & 122 & 160 & 0 & 4 & 3,402 \\
\hline & 9.78 & 5.16 & 17.49 & 0 & 2.67 & 9.64 \\
\hline \multirow{2}{*}{$\begin{array}{l}\text { Member of an agriculture } \\
\text { association }\end{array}$} & 8 & 1 & 0 & 0 & 0 & 9 \\
\hline & 0.03 & 0.04 & 0 & 0 & 0 & 0.03 \\
\hline \multirow[b]{2}{*}{ Family help } & 627 & 45 & 12 & 0 & 3 & 687 \\
\hline & 1.97 & 1.9 & 1.31 & 0 & 2 & 1.95 \\
\hline \multirow[b]{2}{*}{ Unemployed } & 1,214 & 92 & 140 & 1 & 4 & 1,451 \\
\hline & 3.81 & 3.89 & 15.3 & 4.35 & 2.67 & 4.11 \\
\hline \multirow[b]{2}{*}{ Retired } & 3,120 & 288 & 31 & 5 & 15 & 3,459 \\
\hline & 9.79 & 12.19 & 3.39 & 21.74 & 10 & 9.8 \\
\hline \multirow[b]{2}{*}{ Pupil } & 1,850 & 167 & 51 & 0 & 5 & 2,073 \\
\hline & 5.81 & 7.07 & 5.57 & 0 & 3.33 & 5.87 \\
\hline \multirow[b]{2}{*}{ Student } & 948 & 66 & 1 & 0 & 7 & 1,022 \\
\hline & 2.98 & 2.79 & 0.11 & 0 & 4.67 & 2.89 \\
\hline \multirow[b]{2}{*}{ House wife } & 2,664 & 230 & 209 & 2 & 24 & 3,129 \\
\hline & 8.36 & 9.73 & 22.84 & 8.7 & 16 & 8.86 \\
\hline \multirow{2}{*}{$\begin{array}{l}\text { Other statute (elderly, } \\
\text { kindergarten, dependent person, } \\
\text { disabled person) }\end{array}$} & 363 & 26 & 55 & 0 & 1 & 445 \\
\hline & 1.14 & 1.1 & 6.01 & 0 & 0.67 & 1.26 \\
\hline \multirow{2}{*}{ Total } & 31,853 & 2,363 & 915 & 23 & 150 & $\begin{array}{r}35,30 \\
4\end{array}$ \\
\hline & 100 & 100 & 100 & 100 & 100 & 100 \\
\hline
\end{tabular}

Source: Household Budget Survey, 2016

If we analyze the occupational status without limiting ourselves to the working age group we can see that $17 \%$ of the Roma are pupils and only $0.07 \%$ are students. We also notice that $8,83 \%$ are pensioners, compared with $37 \%$ of Romanians, and $17 \%$ are house wives, compared to $6 \%$ of Romanians. We note that the percentage of people with a different status of dependent person is $15 \%$ for the Roma as opposed to $4 \%$ for the Romanians.

Table 10. Occupational status in the last year by nationality

\begin{tabular}{|c|c|c|c|c|c|c|}
\hline $\begin{array}{l}\text { Occupational status in } \\
\text { the last } 12 \text { months }\end{array}$ & Romanian & Hungarian & Roma & Germans & Others & Total \\
\hline \multirow[b]{2}{*}{ Employee } & 16,705 & 1,206 & 141 & 11 & 75 & 18,138 \\
\hline & 30.5 & 29.34 & 10.21 & 17.19 & 31.51 & 29.95 \\
\hline \multirow[b]{2}{*}{ Entrepreneur } & 102 & 5 & 2 & 0 & 6 & 115 \\
\hline & 0.19 & 0.12 & 0.14 & 0 & 2.52 & 0.19 \\
\hline \multirow{2}{*}{$\begin{array}{l}\text { Self-employed worker } \\
\text { in other activities than } \\
\text { agriculture }\end{array}$} & 1,515 & 131 & 120 & 5 & 7 & 1,778 \\
\hline & 2.77 & 3.19 & 8.69 & 7.81 & 2.94 & 2.94 \\
\hline \multirow{2}{*}{$\begin{array}{l}\text { Member of a non- } \\
\text { agricultural cooperative }\end{array}$} & 11 & 8 & 3 & 0 & 0 & 22 \\
\hline & 0.02 & 0.19 & 0.22 & 0 & 0 & 0.04 \\
\hline
\end{tabular}




\begin{tabular}{|c|c|c|c|c|c|c|}
\hline $\begin{array}{l}\text { Occupational status in } \\
\text { the last } 12 \text { months }\end{array}$ & Romanian & Hungarian & Roma & Germans & Others & Total \\
\hline \multirow{2}{*}{$\begin{array}{l}\text { Own account worker } \\
\text { in agriculture }\end{array}$} & 3,413 & 133 & 165 & 0 & 4 & 3,715 \\
\hline & 6.23 & 3.24 & 11.95 & 0 & 1.68 & 6.13 \\
\hline \multirow{2}{*}{$\begin{array}{l}\text { Member of an } \\
\text { agriculture association }\end{array}$} & 8 & 1 & 0 & 0 & 0 & 9 \\
\hline & 0.01 & 0.02 & 0 & 0 & 0 & 0.01 \\
\hline \multirow[b]{2}{*}{ Family help } & 678 & 46 & 12 & 0 & 3 & 739 \\
\hline & 1.24 & 1.12 & 0.87 & 0 & 1.26 & 1.22 \\
\hline \multirow[b]{2}{*}{ Unemployed } & 1,230 & 95 & 141 & 2 & 4 & 1,472 \\
\hline & 2.25 & 2.31 & 10.21 & 3.13 & 1.68 & 2.43 \\
\hline \multirow[b]{2}{*}{ Retired } & 20,098 & 1,626 & 122 & 40 & 87 & 21,973 \\
\hline & 36.69 & 39.55 & 8.83 & 62.5 & 36.55 & 36.28 \\
\hline \multirow[b]{2}{*}{ Pupil } & 4,817 & 388 & 241 & 3 & 17 & 5,466 \\
\hline & 8.79 & 9.44 & 17.45 & 4.69 & 7.14 & 9.02 \\
\hline \multirow[b]{2}{*}{ Student } & 948 & 66 & 1 & 0 & 7 & 1,022 \\
\hline & 1.73 & 1.61 & 0.07 & 0 & 2.94 & 1.69 \\
\hline \multirow[b]{2}{*}{ House wife } & 3,087 & 257 & 228 & 2 & 26 & 3,600 \\
\hline & 5.64 & 6.25 & 16.51 & 3.13 & 10.92 & 5.94 \\
\hline \multirow{2}{*}{$\begin{array}{l}\text { Other statute (elderly, } \\
\text { kindergarten, dependent } \\
\text { person, disabled person, } \\
\text { born in the month of } \\
\text { the research }\end{array}$} & 2,163 & 149 & 205 & 1 & 2 & 2,520 \\
\hline & 3.95 & 3.62 & 14.84 & 1.56 & 0.84 & 4.16 \\
\hline \multirow[b]{2}{*}{ Total } & 54,775 & 4,111 & 1,381 & 64 & 238 & 60,569 \\
\hline & 100 & 100 & 100 & 100 & 100 & 100 \\
\hline
\end{tabular}

Source: Household Budget Survey, 2016

As can be seen in the table below, only $17 \%$ of Roma men are employed, $20 \%$ are selfemployed workers in other fields than agriculture, $22 \%$ work are self-employed workers in agriculture, $20 \%$ are unemployed and about $6 \%$ of men are either pupils or students. If we analyze the professional structure of women able to work, $46 \%$ of women are housewives, $13 \%$ are employed, $3 \%$ are self-employed in non-agricultural fields, $13 \%$ are self-employed in agriculture, $2 \%$ are family workers, $10 \%$ are unemployed, $3 \%$ are retired, $5 \%$ are students or $5 \%$ are other categories of dependents. Therefore, almost half of the women are housewives, which means that the structure of the traditional family has been maintained.

Table 11. Roma occupational status for people aged 15-61, by nationality and gender

\begin{tabular}{|l|r|r|r|}
\hline \multicolumn{1}{|c|}{$\begin{array}{c}\text { Roma occupational status } \\
\text { in the last 12 months }\end{array}$} & Men & Women & \multicolumn{1}{c|}{ Total } \\
\hline For people aged 15-61 years & & & \\
\hline \multirow{3}{*}{ Employee } & 83 & 57 & 140 \\
\hline \multirow{3}{*}{ Entrepreneur } & 17.93 & 12.61 & 15.3 \\
\cline { 2 - 4 } & 2 & 0 & 2 \\
\hline
\end{tabular}




\begin{tabular}{|c|c|c|c|}
\hline $\begin{array}{l}\text { Roma occupational status } \\
\text { in the last } 12 \text { months }\end{array}$ & Men & Women & Total \\
\hline \multirow[b]{2}{*}{ Self-employed worker in other fields than agriculture } & 96 & 15 & 111 \\
\hline & 20.73 & 3.32 & 12.13 \\
\hline \multirow[b]{2}{*}{ Member of a non-agriculture cooperative } & 3 & 0 & 3 \\
\hline & 0.65 & 0 & 0.33 \\
\hline \multirow[b]{2}{*}{ Self-employed worker in agriculture } & 103 & 57 & 160 \\
\hline & 22.25 & 12.61 & 17.49 \\
\hline \multirow[b]{2}{*}{ Family help } & 3 & 9 & 12 \\
\hline & 0.65 & 1.99 & 1.31 \\
\hline \multirow[b]{2}{*}{ Unemployed } & 95 & 45 & 140 \\
\hline & 20.52 & 9.96 & 15.3 \\
\hline \multirow[b]{2}{*}{ Retired } & 17 & 14 & 31 \\
\hline & 3.67 & 3.1 & 3.39 \\
\hline \multirow[b]{2}{*}{ Pupil } & 30 & 21 & 51 \\
\hline & 6.48 & 4.65 & 5.57 \\
\hline \multirow[b]{2}{*}{ Student } & 0 & 1 & 1 \\
\hline & 0 & 0.22 & 0.11 \\
\hline \multirow[b]{2}{*}{ Housewife } & 0 & 209 & 209 \\
\hline & 0 & 46.24 & 22.84 \\
\hline \multirow[t]{2}{*}{ Other statute (dependent person) } & 31 & 24 & 55 \\
\hline & 6.7 & 5.31 & 6.01 \\
\hline \multirow[b]{2}{*}{ Total } & 463 & 452 & 915 \\
\hline & 100 & 100 & 100 \\
\hline
\end{tabular}

Source: Household Budget Survey, 2016

Of the working men in Romania, about $57 \%$ are employees, $7 \%$ work in other fields than agriculture, $11 \%$ work on their own in agriculture, $5 \%$ are unemployed, $8 \%$ are retired, $9 \%$ are pupils or students $1 \%$ falls into another category of dependents in the last 12 months. Of the Romanian women aged $15-61,46 \%$ are employees, $8 \%$ are selfemployed in agriculture, $3 \%$ are family workers, $12 \%$ are unemployed, $12 \%$ are retired, $9 \%$ are pupils, $16 \%$ are housewives and $1 \%$ fall into another category of dependents in the last 12 months.

Table 12. Romanian Occupational status for people aged 15-61, by gender

\begin{tabular}{|c|c|c|c|}
\hline Romanian Occupational status in the last 12 months & Men & Women & Total \\
\hline \multicolumn{4}{|l|}{ Forpersons with the age 15-61 years } \\
\hline \multirow[b]{2}{*}{ Employee } & 8,931 & 7,437 & 16,368 \\
\hline & 57.02 & 45.94 & 51.39 \\
\hline \multirow[b]{2}{*}{ Entrepreneur } & 58 & 26 & 84 \\
\hline & 0.37 & 0.16 & 0.26 \\
\hline \multirow[b]{2}{*}{ Self-employed worker in other than agriculture fields } & 1,084 & 396 & 1,480 \\
\hline & 6.92 & 2.45 & 4.65 \\
\hline \multirow[b]{2}{*}{ Member of a non-agriculture cooperative } & 4 & 7 & 11 \\
\hline & 0.03 & 0.04 & 0.03 \\
\hline
\end{tabular}




\begin{tabular}{|l|r|r|r|}
\hline Romanian Occupational status in the last 12 months & \multicolumn{1}{|c|}{ Men } & Women & Total \\
\hline \multirow{3}{*}{ Self-employed worker in agriculture } & 1,741 & 1,375 & 3,116 \\
\cline { 2 - 4 } Member of an agricultural association & 11.11 & 8.49 & 9.78 \\
\hline \multirow{3}{*}{ Family help } & 5 & 3 & 8 \\
\hline \multirow{3}{*}{ Unemployed } & 0.03 & 0.02 & 0.03 \\
\hline \multirow{3}{*}{ Retired } & 159 & 468 & 627 \\
\hline \multirow{3}{*}{ Pupil } & 1.02 & 2.89 & 1.97 \\
\hline \multirow{3}{*}{ Student } & 823 & 391 & 1,214 \\
\hline \multirow{3}{*}{ House wife } & 5.25 & 2.42 & 3.81 \\
\hline \multirow{3}{*}{ Other statute (dependent) } & 1,231 & 1,889 & 3,120 \\
\cline { 2 - 4 } & 7.86 & 11.67 & 9.79 \\
\hline Total & 951 & 899 & 1,850 \\
\hline & 6.07 & 5.55 & 5.81 \\
\cline { 2 - 4 } & 469 & 479 & 948 \\
\cline { 2 - 4 } & 2.99 & 2.96 & 2.98 \\
\hline
\end{tabular}

Source: Household Budget Survey, 2016

Next, we use the data distribution in rural and urban areas as well as by gender and nationality. Among the Roma, we see that in urban areas, about one quarter of the men are employed, $23 \%$ work on their own in non-agricultural (industrial or services), $7 \%$ self-employed in agriculture, $27 \%$ are unemployed, $5 \%$ are retired, $6 \%$ are pupils or students and $5 \%$ are in other categories of dependents. Among women in urban areas, only $18 \%$ are employed, $4 \%$ work on their own in non-agricultural fields, $3 \%$ work on their own in agriculture, $14 \%$ are unemployed, 5\% are retired, 5\% students and $49 \%$ are housewives. This means that although they are in urban areas, due to limited opportunities for employment, discrimination or low educational level, the structure of the traditional Roma family is preserved.

Table 13. Occupational status for people aged 15-61, in the urban areas by gender

\begin{tabular}{|l|r|r|r|}
\hline $\begin{array}{c}\text { Urban Occupational status } \\
\text { in the last 12 months }\end{array}$ & \multicolumn{1}{c|}{ Men } & Women & \multicolumn{1}{c|}{ Total } \\
\hline People aged 15 to 61 years & 42 & 31 & 73 \\
\hline \multirow{3}{*}{ Employee } & 25.3 & 18.13 & 21.66 \\
\hline \multirow{3}{*}{ Entrepreneur } & 1 & 0 & 1 \\
\hline \multirow{2}{*}{ Self-employed worker in non-agricultural fields } & 0.6 & 0 & 0.3 \\
\cline { 2 - 5 } & 38 & 6 & 44 \\
\hline
\end{tabular}




\begin{tabular}{|l|r|r|r|}
\hline $\begin{array}{c}\text { Urban Occupational status } \\
\text { in the last 12 months }\end{array}$ & \multicolumn{1}{c|}{ Men } & \multicolumn{1}{c|}{ Women } & \multicolumn{1}{c|}{ Total } \\
\hline \multirow{3}{*}{ Self-employed worker in agriculture } & 12 & 5 & 17 \\
\hline \multirow{3}{*}{ Family help } & 7.23 & 2.92 & 5.04 \\
\hline \multirow{3}{*}{ Unemployed } & 1 & 0 & 1 \\
\hline \multirow{3}{*}{ Retired } & 0.6 & 0 & 0.3 \\
\hline \multirow{3}{*}{ Pupil } & 44 & 24 & 68 \\
\hline \multirow{3}{*}{ Student } & 26.51 & 14.04 & 20.18 \\
\hline \multirow{3}{*}{ Housewives } & 9 & 9 & 18 \\
\hline \multirow{3}{*}{ Alt statute (dependent) } & 5.42 & 5.26 & 5.34 \\
\hline \multirow{3}{*}{ Total } & 10 & 7 & 17 \\
\cline { 2 - 4 } & 6.02 & 4.09 & 5.04 \\
\cline { 2 - 4 } & 0 & 1 & 1 \\
\cline { 2 - 4 } & 0 & 0.58 & 0.3 \\
\cline { 2 - 4 } & 0 & 83 & 83 \\
\cline { 2 - 4 } & 0 & 48.54 & 24.63 \\
\hline
\end{tabular}

Source: Household Budget Survey, 2016

In rural areas, a lower percentage of men and women are employed, about $14 \%$ of men are employed, $20 \%$ work as self-employed , 31\% of men work in agriculture, $17 \%$ of men are unemployed, a lower percentage than in urban areas, 3\% are retired, $7 \%$ are students. Among women in rural areas, 9\% are employed, 3\% work on their own in non-agricultural fields, $19 \%$ work on their own in agriculture, $3 \%$ are family workers, $7 \%$ are unemployed, low compared to urban areas, $2 \%$ are retirement, $5 \%$ are female and about $45 \%$ are housewives. The data shows that, irrespective of urban or rural areas, about half of Roma women are housewives, which means maintaining the structure of the traditional Roma family.

Table 14. Occupational status for people aged 15-61, living in the rural area for Roma by gender

\begin{tabular}{|c|c|c|c|}
\hline Rural Roma Occupational statute in the last 12 months & Men & Women & Total \\
\hline \multicolumn{4}{|l|}{ Persons aged 15-61 } \\
\hline \multirow[b]{2}{*}{ Employee } & 41 & 26 & 67 \\
\hline & 13.8 & 9.25 & 11.59 \\
\hline \multirow[b]{2}{*}{ Entrepreneur } & 1 & 0 & 1 \\
\hline & 0.34 & 0 & 0.17 \\
\hline \multirow[b]{2}{*}{ Self-employed in other than agriculture } & 58 & 9 & 67 \\
\hline & 19.53 & 3.2 & 11.59 \\
\hline \multirow[b]{2}{*}{ Member of a non-agriculture cooperative } & 3 & 0 & 3 \\
\hline & 1.01 & 0 & 0.52 \\
\hline \multirow[b]{2}{*}{ Self-employed in agriculture } & 91 & 52 & 143 \\
\hline & 30.64 & 18.51 & 24.74 \\
\hline
\end{tabular}




\begin{tabular}{|l|r|r|r|}
\hline Rural Roma Occupational statute in the last 12 months & Men & Women & Total \\
\hline \multirow{3}{*}{ Family help } & 2 & 9 & 11 \\
\hline \multirow{3}{*}{ Unemployed } & 0.67 & 3.2 & 1.9 \\
\hline \multirow{3}{*}{ Retired } & 51 & 21 & 72 \\
\hline \multirow{3}{*}{ Pupil } & 17.17 & 7.47 & 12.46 \\
\hline \multirow{3}{*}{ Housewife } & 8 & 5 & 13 \\
\hline \multirow{3}{*}{ Other statute (dependent person) } & 2.69 & 1.78 & 2.25 \\
\hline \multirow{3}{*}{ Total } & 20 & 14 & 34 \\
\cline { 2 - 4 } & 6.73 & 4.98 & 5.88 \\
\hline
\end{tabular}

Source: Household Budget Survey, 2016

If we look at the situation of poverty, about $75 \%$ of Roma are at risk of poverty, compared to $20 \%$ for Romanians. There is a higher tendency towards Roma poverty, which is due to a higher likelihood of self-employed work, either in agriculture or in non-agricultural sectors, in combination with a traditional family structure, where women are housewives and there is a large number of elders and children in need of care. In addition, about $69 \%$ of Roma working are poor, compared to $17 \%$ of Romanians in this situation. As a result of a precarious educational situation, working fails to bring the Roma out of poverty. Poverty persists regardless of the professional situation, with only $6 \%$ of Roma getting out of poverty by working. Of the Roma who do not work, $75 \%$ are poor, compared to $21 \%$ of the Romanians in the same situation. Therefore, the inequalities between the Roma and the Romanians appear to be manifested on several levels. Approximately 44\% of Roma work, compared with only $49 \%$ of Romanians. If among the Romanians the main reasons for not having a job are either retired or having a socially assisted status, with about $35 \%$ of Romanians having the same reasons, there is a strong tendency among the Roma to motivate their lack of a job by the fact that women are housewives ( $21 \%$ of Roma), in combination with a larger family of dependent children and elderly people.

Table 15. Poverty by nationality

\begin{tabular}{|l|r|r|r|r|r|r|}
\hline Poverty & Romanian & Hungarian & Roma & Germans & Others & Total \\
\hline \multirow{3}{*}{ No } & 44,040 & 3,372 & 344 & 60 & 199 & 48,015 \\
\cline { 2 - 7 } & 80.4 & 82.02 & 24.91 & 93.75 & 83.61 & 79.27 \\
\hline \multirow{3}{*}{ Yes } & 10,735 & 739 & 1,037 & 4 & 39 & 12,554 \\
\hline \multirow{3}{*}{ Total } & 19.6 & 17.98 & 75.09 & 6.25 & 16.39 & 20.73 \\
\cline { 2 - 7 } & 54,775 & 4,111 & 1,381 & 64 & 238 & 60,569 \\
\hline
\end{tabular}

Source: Household Budget Survey, 2016 
Table 16. Poverty among the working poor by nationality

\begin{tabular}{|l|r|r|r|r|r|r|}
\hline $\begin{array}{c}\text { Poverty among the } \\
\text { working poor }\end{array}$ & Romanian & Hungarian & Roma & Germans & Others & \multicolumn{1}{c|}{ Total } \\
\hline No & 20,405 & 1,358 & 145 & 16 & 89 & 22,013 \\
\hline & 82.95 & 87.44 & 31.45 & 100 & 93.68 & 82.37 \\
\hline Yes & 4,193 & 195 & 316 & 0 & 6 & 4,710 \\
\hline & 17.05 & 12.56 & 68.55 & 0 & 6.32 & 17.63 \\
\hline Total & 24,598 & 1,553 & 461 & 16 & 95 & 26,723 \\
\hline & 100 & 100 & 100 & 100 & 100 & 100 \\
\hline
\end{tabular}

Source: ABF 2016, own calculations

Table 17. Poverty among those that do not work by nationality

\begin{tabular}{|l|r|r|r|r|r|r|}
\hline $\begin{array}{c}\text { Poverty among } \\
\text { those .. }\end{array}$ & Romanian & Hungarian & Roma & Germans & Others & \multicolumn{1}{c|}{ Total } \\
\hline \multirow{3}{*}{ No } & 20,225 & 1,742 & 150 & 40 & 101 & 22,258 \\
\cline { 2 - 7 } & 79.31 & 78.43 & 25.17 & 90.91 & 77.69 & 78.12 \\
\hline \multirow{3}{*}{ Yes } & 5,276 & 479 & 446 & 4 & 29 & 6,234 \\
\cline { 2 - 7 } Total & 20.69 & 21.57 & 74.83 & 9.09 & 22.31 & 21.88 \\
\cline { 2 - 7 } & 25,501 & 2,221 & 596 & 44 & 130 & 28,492 \\
\hline
\end{tabular}

Source: Household Budget Survey, 2016

Table 18. Reasons for absence from work

\begin{tabular}{|c|c|c|c|c|c|c|}
\hline $\begin{array}{l}\text { Reasons for missing from } \\
\text { work }\end{array}$ & Romanian & Hungarian & Roma & Germans & Others & Total \\
\hline \multirow[b]{2}{*}{$\mathrm{He} /$ she worked } & 24,598 & 1,553 & 461 & 16 & 95 & 26,723 \\
\hline & 49.1 & 41.15 & 43.61 & 26.67 & 42.22 & 48.4 \\
\hline \multirow[b]{2}{*}{ Absent from work } & 308 & 31 & 2 & 0 & 0 & 341 \\
\hline & 0.61 & 0.82 & 0.19 & 0 & 0 & 0.62 \\
\hline \multirow{2}{*}{$\begin{array}{l}\mathrm{He} / \text { she found work and will } \\
\text { start activity subsequently }\end{array}$} & 14 & 1 & 1 & 0 & 0 & 16 \\
\hline & 0.03 & 0.03 & 0.09 & 0 & 0 & 0.03 \\
\hline \multirow{2}{*}{$\begin{array}{l}\text { Is looking for work/ } \\
\text { unemployed }\end{array}$} & 1,230 & 115 & 136 & 2 & 4 & 1,487 \\
\hline & 2.46 & 3.05 & 12.87 & 3.33 & 1.78 & 2.69 \\
\hline \multirow[b]{2}{*}{ Pupils or students } & 2,709 & 226 & 48 & 0 & 12 & 2,995 \\
\hline & 5.41 & 5.99 & 4.54 & 0 & 5.33 & 5.42 \\
\hline \multirow{2}{*}{$\begin{array}{l}\text { Retired - age limit, illness, } \\
\text { invalidity, survivor, social } \\
\text { assistance }\end{array}$} & 17,743 & 1,560 & 119 & 40 & 87 & 19,549 \\
\hline & 35.42 & 41.34 & 11.26 & 66.67 & 38.67 & 35.41 \\
\hline \multirow[b]{2}{*}{ Housewives } & 3,063 & 255 & 225 & 2 & 26 & 3,571 \\
\hline & 6.11 & 6.76 & 21.29 & 3.33 & 11.56 & 6.47 \\
\hline \multirow[b]{2}{*}{ Other reasons } & 434 & 33 & 65 & 0 & 1 & 533 \\
\hline & 0.87 & 0.87 & 6.15 & 0 & 0.44 & 0.97 \\
\hline \multirow[b]{2}{*}{ Total } & 50,099 & 3,774 & 1,057 & 60 & 225 & 55,215 \\
\hline & 100 & 100 & 100 & 100 & 100 & 100 \\
\hline
\end{tabular}

Source: Household Budget Survey, 2016 
If we analyze the professional situation of people aged 15-61 we can see that about $51 \%$ of Roma do not work, compared to $31 \%$ of Romanians. Among the Roma, there is a greater tendency not to work, and if they do work, they work on their own account either in agriculture or in non-agricultural fields. As a result of the low level of school education, Roma tend to work on their own account. Out of the workforce, about $15 \%$ of Roma are employed, compared with $51 \%$ of Romanians, $15 \%$ work on their own in non-agricultural fields, compared to only $5 \%$ for Romanians, $17 \%$ of Roma work on their own in agriculture, compared to $10 \%$ of Romanians, and about $2 \%$ work to support their family, 3\% in the case of Romanians.

Table 19. Roma the educational level attained by generations

\begin{tabular}{|c|c|c|c|c|c|c|c|c|c|c|c|}
\hline $\begin{array}{c}\text { Roma the } \\
\text { educational level } \\
\text { attained }\end{array}$ & $\begin{array}{c}<7 \\
\text { years }\end{array}$ & $\begin{array}{r}7-14 \\
\text { years }\end{array}$ & $\begin{array}{l}15-18 \\
\text { years }\end{array}$ & $\begin{array}{l}19-24 \\
\text { years }\end{array}$ & $\begin{array}{l}25-30 \\
\text { years }\end{array}$ & $\begin{array}{l}31-40 \\
\text { years }\end{array}$ & $\begin{array}{l}41-50 \\
\text { years }\end{array}$ & $\begin{array}{c}51-60 \\
\text { years }\end{array}$ & $61-65$ & $>=66$ & Total \\
\hline \multirow[b]{2}{*}{ Without any school } & 144 & 23 & 2 & 6 & 8 & 16 & 20 & 12 & 5 & 15 & 251 \\
\hline & 76.19 & 11.17 & 2.67 & 4.58 & 6.9 & 7.51 & 10.31 & 9.09 & 9.8 & 20.27 & 18.18 \\
\hline \multirow[b]{2}{*}{ Kindergarten } & 45 & 17 & 5 & 3 & 2 & 1 & 0 & 1 & 0 & 2 & 76 \\
\hline & 23.81 & 8.25 & 6.67 & 2.29 & 1.72 & 0.47 & 0 & 0.76 & 0 & 2.7 & 5.5 \\
\hline \multirow{2}{*}{$\begin{array}{l}\text { Primary school } \\
\text { (classes } 0-4)\end{array}$} & 0 & 110 & 20 & 42 & 41 & 66 & 73 & 56 & 23 & 46 & 477 \\
\hline & 0 & 53.4 & 26.67 & 32.06 & 35.34 & 30.99 & 37.63 & 42.42 & 45.1 & 62.16 & 34.54 \\
\hline \multirow{2}{*}{$\begin{array}{l}\text { Gymnasium (classes } \\
5-8 \text { ) }\end{array}$} & 0 & 50 & 28 & 68 & 55 & 102 & 80 & 48 & 19 & 10 & 460 \\
\hline & 0 & 24.27 & 37.33 & 51.91 & 47.41 & 47.89 & 41.24 & 36.36 & 37.25 & 13.51 & 33.31 \\
\hline \multirow{2}{*}{$\begin{array}{l}\text { Professional school } \\
\text { (vocational school) }\end{array}$} & 0 & 0 & 4 & 2 & 5 & 10 & 12 & 9 & 3 & 1 & 46 \\
\hline & 0 & 0 & 5.33 & 1.53 & 4.31 & 4.69 & 6.19 & 6.82 & 5.88 & 1.35 & 3.33 \\
\hline \multirow{2}{*}{$\begin{array}{l}\text { Highschool (classes } 9 \\
\text { or } 10 \text { ) }\end{array}$} & 0 & 6 & 7 & 6 & 4 & 11 & 4 & 2 & 0 & 0 & 40 \\
\hline & 0 & 2.91 & 9.33 & 4.58 & 3.45 & 5.16 & 2.06 & 1.52 & 0 & 0 & 2.9 \\
\hline \multirow{2}{*}{$\begin{array}{l}\text { Highschool (class 11- } \\
12 / 13 \text { ) }\end{array}$} & 0 & 0 & 9 & 4 & 0 & 5 & 3 & 4 & 0 & 0 & 25 \\
\hline & 0 & 0 & 12 & 3.05 & 0 & 2.35 & 1.55 & 3.03 & 0 & 0 & 1.81 \\
\hline \multirow{2}{*}{$\begin{array}{l}\text { Post high-school } \\
\text { technical school }\end{array}$} & 0 & 0 & 0 & 0 & 1 & 1 & 0 & 0 & 1 & 0 & 3 \\
\hline & 0 & 0 & 0 & 0 & 0.86 & 0.47 & 0 & 0 & 1.96 & 0 & 0.22 \\
\hline \multirow[b]{2}{*}{ University III (PhD) } & 0 & 0 & 0 & 0 & 0 & 0 & 1 & 0 & 0 & 0 & 1 \\
\hline & 0 & 0 & 0 & 0 & 0 & 0 & 0.52 & 0 & 0 & 0 & 0.07 \\
\hline \multirow{2}{*}{$\begin{array}{l}\text { University long } \\
\text { duration (4-6 years) }\end{array}$} & 0 & 0 & 0 & 0 & 0 & 1 & 1 & 0 & 0 & 0 & 2 \\
\hline & 0 & 0 & 0 & 0 & 0 & 0.47 & 0.52 & 0 & 0 & 0 & 0.14 \\
\hline \multirow[b]{2}{*}{ Total } & 189 & 206 & 75 & 131 & 116 & 213 & 194 & 132 & 51 & 74 & 1,381 \\
\hline & 100 & 100 & 100 & 100 & 100 & 100 & 100 & 100 & 100 & 100 & 100 \\
\hline
\end{tabular}

Source: Household Budget Survey, 2016

Table 20. Romanian educational level attained y generations

\begin{tabular}{|c|c|c|c|c|c|c|c|c|c|c|c|}
\hline $\begin{array}{c}\text { Romanian educational } \\
\text { level attained }\end{array}$ & $\begin{array}{c}<7 \\
\text { years }\end{array}$ & $\begin{array}{r}7-14 \\
\text { years }\end{array}$ & $\begin{array}{c}15-18 \\
\text { years }\end{array}$ & $\begin{array}{c}19-24 \\
\text { years }\end{array}$ & $\begin{array}{l}25-30 \\
\text { years }\end{array}$ & $\begin{array}{l}31-40 \\
\text { years }\end{array}$ & $\begin{array}{l}41-50 \\
\text { years }\end{array}$ & $\begin{array}{c}51-60 \\
\text { years }\end{array}$ & $61-65$ & $>=66$ & Total \\
\hline \multirow{2}{*}{$\begin{array}{l}\text { Without any } \\
\text { completed school }\end{array}$} & 1,894 & 116 & 4 & 7 & 12 & 29 & 18 & 14 & 3 & 102 & 2,199 \\
\hline & 67.86 & 3.52 & 0.23 & 0.29 & 0.39 & 0.39 & 0.21 & 0.16 & 0.05 & 0.96 & 4.01 \\
\hline \multirow[b]{2}{*}{ Kindergarten } & 897 & 300 & 0 & 0 & 0 & 4 & 3 & 4 & 2 & 42 & 1,252 \\
\hline & 32.14 & 9.11 & 0 & 0 & 0 & 0.05 & 0.03 & 0.04 & 0.03 & 0.4 & 2.29 \\
\hline \multirow{2}{*}{$\begin{array}{l}\text { Primary school (class } \\
0-4 \text { ) }\end{array}$} & 0 & 1,452 & 12 & 30 & 48 & 131 & 129 & 326 & 387 & 3,244 & 5,759 \\
\hline & 0 & 44.11 & 0.7 & 1.26 & 1.58 & 1.74 & 1.48 & 3.63 & 6.72 & 30.62 & 10.51 \\
\hline
\end{tabular}


Dimensions of multiple deprivation in Roma population | 35

\begin{tabular}{|c|c|c|c|c|c|c|c|c|c|c|c|}
\hline $\begin{array}{c}\text { Romanian educational } \\
\text { level attained }\end{array}$ & $\begin{array}{c}<7 \\
\text { years }\end{array}$ & $\begin{array}{r}7-14 \\
\text { years }\end{array}$ & $\begin{array}{c}15-18 \\
\text { years }\end{array}$ & $\begin{array}{l}19-24 \\
\text { years }\end{array}$ & $\begin{array}{l}25-30 \\
\text { years }\end{array}$ & $\begin{array}{l}31-40 \\
\text { years }\end{array}$ & $\begin{array}{l}41-50 \\
\text { years }\end{array}$ & $\begin{array}{c}51-60 \\
\text { years }\end{array}$ & $61-65$ & $>=66$ & Total \\
\hline \multirow{2}{*}{$\begin{array}{l}\text { Gymnasium } \\
\text { (class 5-8) }\end{array}$} & 0 & 1,177 & 290 & 251 & 370 & 1,030 & 1,013 & 1,811 & 1,517 & 3,431 & 10,890 \\
\hline & 0 & 35.75 & 16.98 & 10.52 & 12.18 & 13.68 & 11.66 & 20.15 & 26.34 & 32.38 & 19.88 \\
\hline \multirow[b]{2}{*}{ Professional school } & 0 & 8 & 96 & 212 & 389 & 1,366 & 2,293 & 2,967 & 1,876 & 1,786 & 10,993 \\
\hline & 0 & 0.24 & 5.62 & 8.89 & 12.8 & 18.14 & 26.39 & 33.02 & 32.57 & 16.86 & 20.07 \\
\hline \multirow{2}{*}{$\begin{array}{l}\text { Highschool (class } 9 \\
\text { or } 10 \text { ) }\end{array}$} & 0 & 239 & 310 & 160 & 178 & 610 & 892 & 569 & 185 & 237 & 3,380 \\
\hline & 0 & 7.26 & 18.15 & 6.71 & 5.86 & 8.1 & 10.27 & 6.33 & 3.21 & 2.24 & 6.17 \\
\hline \multirow{2}{*}{$\begin{array}{l}\text { Highschool (class } 11 \\
\text { or } 12 / 13 \text { ) }\end{array}$} & 0 & 0 & 972 & 1,121 & 1,009 & 2,439 & 2,857 & 2,069 & 949 & 815 & 12,231 \\
\hline & 0 & 0 & 56.91 & 46.98 & 33.21 & 32.39 & 32.88 & 23.02 & 16.48 & 7.69 & 22.33 \\
\hline \multirow{2}{*}{$\begin{array}{l}\text { Post high-school } \\
\text { vocational eduction/ } \\
\text { technical school }\end{array}$} & 0 & 0 & 9 & 117 & 216 & 484 & 436 & 439 & 445 & 463 & 2,609 \\
\hline & 0 & 0 & 0.53 & 4.9 & 7.11 & 6.43 & 5.02 & 4.89 & 7.73 & 4.37 & 4.76 \\
\hline \multirow{2}{*}{$\begin{array}{l}\text { University I (BA } \\
\text { studies) }\end{array}$} & $\overline{0}$ & 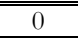 & 13 & 183 & 143 & 124 & 91 & 12 & 0 & 0 & 5666 \\
\hline & 0 & 0 & 0.76 & 7.67 & 4.71 & 1.65 & 1.05 & 0.13 & 0 & 0 & 1.03 \\
\hline \multirow{2}{*}{$\begin{array}{l}\text { University II (MA } \\
\text { studies) }\end{array}$} & 0 & 0 & 0 & 44 & 45 & 49 & 22 & 7 & 5 & 0 & 172 \\
\hline & 0 & 0 & 0 & 1.84 & 1.48 & 0.65 & 0.25 & 0.08 & 0.09 & 0 & 0.31 \\
\hline \multirow{2}{*}{$\begin{array}{l}\text { University III (PhD } \\
\text { level) }\end{array}$} & 0 & 0 & 0 & 2 & 15 & 38 & 20 & 10 & 3 & 4 & 92 \\
\hline & 0 & 0 & 0 & 0.08 & 0.49 & 0.5 & 0.23 & 0.11 & 0.05 & 0.04 & 0.17 \\
\hline \multirow{2}{*}{$\begin{array}{l}\text { University long } \\
\text { duration (4-6 years) } \\
\end{array}$} & 0 & 0 & 2 & 246 & 589 & 1,191 & 888 & 745 & 383 & 459 & 4,503 \\
\hline & 0 & 0 & 0.12 & 10.31 & 19.39 & 15.81 & 10.22 & 8.29 & 6.65 & 4.33 & 8.22 \\
\hline \multirow[b]{2}{*}{ Post-university } & 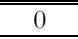 & 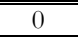 & 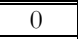 & 13 & 23 & 35 & 24 & 9 & 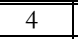 & 3 & 1111 \\
\hline & 0 & 0 & 0 & 0.54 & 0.76 & 0.46 & 0.28 & 0.1 & 0.07 & 0.03 & 0.2 \\
\hline \multirow[b]{2}{*}{$\mathrm{PhD} /$ postdoc } & 0 & 0 & 0 & 0 & 1 & 1 & 2 & 4 & 1 & 9 & 18 \\
\hline & 0 & 0 & 0 & 0 & 0.03 & 0.01 & 0.02 & 0.04 & 0.02 & 0.08 & 0.03 \\
\hline \multirow[b]{2}{*}{ Total } & 2,791 & 3,292 & 1,708 & 2,386 & 3,038 & 7,531 & 8,688 & 8,986 & 5,760 & 10,595 & 54,775 \\
\hline & 100 & 100 & 100 & 100 & 100 & 100 & 100 & 100 & 100 & 100 & 100 \\
\hline
\end{tabular}

Source: Household Budget Survey, 2016

Among the Roma, about $7 \%$ of people aged $14-18$ work, compared with only $4 \%$ among Romanians. The figure grows normally with age and decreases after 50 years. For people aged between 25 and 30, about 50\% of Roma work, compared with $77 \%$ for Romanians. Therefore, there is a lower tendency to work.

Table 21. Roma work by generations

\begin{tabular}{|c|c|c|c|c|c|c|c|c|c|}
\hline $\begin{array}{c}\text { Roma } \\
\text { Work/Years }\end{array}$ & 14-18 & $19-24$ & $25-30$ & $31-40$ & $41-50$ & $51-60$ & $61-65$ & $65>$ & Total \\
\hline \multirow[b]{2}{*}{ Yes } & 6 & 56 & 70 & 115 & 112 & 80 & 17 & 5 & 461 \\
\hline & 7.14 & 47.46 & 50.36 & 58.08 & 55.45 & 48.78 & 34 & 4.9 & 43.61 \\
\hline \multirow[b]{2}{*}{ No } & 78 & 62 & 69 & 83 & 90 & 84 & 33 & 97 & 596 \\
\hline & 92.86 & 52.54 & 49.64 & 41.92 & 44.55 & 51.22 & 66 & 95.1 & 56.39 \\
\hline
\end{tabular}

Source: Household Budget Survey, 2016 
Table 22. Romanian work by generations

\begin{tabular}{|l|r|r|r|r|r|r|r|r|r|}
\hline $\begin{array}{c}\text { Romanian } \\
\text { Work }\end{array}$ & $\mathbf{1 4 - 1 8}$ & $\mathbf{1 9 - 2 4}$ & $\mathbf{2 5 - 3 0}$ & $\mathbf{3 1 - 4 0}$ & $\mathbf{4 1 - 5 0}$ & $\mathbf{5 1 - 6 0}$ & $\mathbf{6 1 - 6 5}$ & $\mathbf{6 5}>$ & Total \\
\hline \multirow{3}{*}{ Yes } & 80 & 1,029 & 2,223 & 5,392 & 7,529 & 5,177 & 1,262 & 1,906 & 24,598 \\
\hline \multirow{3}{*}{ No } & 4.44 & 42.28 & 77.48 & 82.7 & 83.28 & 63.27 & 23.98 & 13.63 & 49.1 \\
\hline \multirow{3}{*}{ Total } & 1,723 & 1,405 & 646 & 1,128 & 1,512 & 3,006 & 4,001 & 12,080 & 25,501 \\
\cline { 2 - 11 }$y$ & 95.56 & 57.72 & 22.52 & 17.3 & 16.72 & 36.73 & 76.02 & 86.37 & 50.9 \\
\cline { 2 - 11 }$y$ & 1,803 & 2,434 & 2,869 & 6,520 & 9,041 & 8,183 & 5,263 & 13,986 & 50,099 \\
\hline
\end{tabular}

Source: Household Budget Survey, 2016

\section{Conclusion}

The current analysis found that Roma have a lower life expectancy than Romanian natives, lower educational attainment, a higher percentage of them are not working, women are more likely to be housewives, men are more likely to be self-employed workers irrespective whether they work in agriculture or non-agricultural domains, and overall Roma have a higher propensity to be at risk of poverty (Cace, Duminica, Preda, 2005; Cace, Preoteasa, Stanescu, Tomescu, 2010). The current article used the Household Budget Survey (HBS) to illustrate some of the multiple deprivation situations Roma face compared to the native Romanians. The article shows that the traditional structure of Roma families is maintained with women more likely to be housewives and take care of children and men more likely to be own account workers. Work is not able to lift the Roma out of poverty because most likely it is done in the rural areas in agriculture or occupations that do not pay much and only $17 \%$ of Roma men are employed. The long term solution for Roma would be to invest in education (Zamfir, Preda, 2002; Duminica, Preda, 2003; Duminica, Cace, 2004; Ionescu, Cace, 2006; Cace, Tomescu, Cojocaru, 2012; Ionescu, Stanescu, 2014); and to create more opportunities for employment in rural areas, re-industrialization could be part of the solution as it would create more and better paid jobs for Roma and as well for Romanians who would not be trapped in subsistence agriculture.

Overall, only $17 \%$ of Roma men are employed, $20 \%$ are self-employed workers in other fields than agriculture, $22 \%$ work are self-employed workers in agriculture, $20 \%$ are unemployed and about $6 \%$ of men are either pupils or students. If we analyze the professional structure of women able to work, $46 \%$ of women are housewives, $13 \%$ are employed, $3 \%$ are self-employed in non-agricultural fields, $13 \%$ are self-employed in agriculture, $2 \%$ are family workers, $10 \%$ are unemployed, $3 \%$ are retired, $5 \%$ are students or $5 \%$ are other categories of dependents.

In rural areas, a lower percentage of men and women are employed, about $14 \%$ of men are employed, $20 \%$ work as self-employed , 31\% of men work in agriculture, $17 \%$ of men are unemployed, a lower percentage than in urban areas, 3\% are retired, $7 \%$ are students. Among women in rural areas, 9\% are employed, 3\% work on their own in non-agricultural fields, $19 \%$ work on their own in agriculture, $3 \%$ are family workers, $7 \%$ are unemployed, low compared to urban areas, $2 \%$ are retirement, $5 \%$ are female and about $45 \%$ are housewives. The data shows that, irrespective of urban or rural 
areas, about half of Roma women are housewives, which means maintaining the structure of the traditional Roma family.

The implications of the current work is that the Household Budget Survey can be used to give an overview of the Roma population and monitor the situation on the long term as it is a rather large survey and applied in a consistent manner over the years.

\section{References}

Cace, S., Duminică, G., Preda, M. (coord.) (2005). Evaluarea programelor pentru comunitățile de romi din România, Agenția de Dezvoltare Comunitară Împreună / UNDP

Cace, S., Preoteasa, A.M., Tomescu, C., Stănescu, S. (2010). Legal si egal pe piața muncii pentru comunitătile de romi. Diagnoza factorilor care influențează nivelul de ocupare la populația de romi. [Legal and illegal on the labour market], Bucureşti: Editura Expert

Cace, S., Tomescu, C., Cojocaru, D. (2012). The role of religion in several Roma communities, Revista de cercetare şi interventie socială, 36: 162-177

Cace, S. (coord.) (2014) Politici de incluriune a romilor in statele membre ale Uniunii Europene, București: Institutul European din România

Duminică, G., Cace, S., Arpinte, D., Ionescu, M., Iova, C., Sali, N. (2004). Politici de incluriune socială pentru grupurile vulnerabile. Cresterea accesului pe piata muncii.

Duminică, G., Preda, M. (2003). Accesul romilor pe piaţa muncii, Editura Cărții de Agribusiness

Ionescu, M., Cace, S.(coord.), Cace, C., Dediu, M., Duminică, G. (2006). Employment Policies for Roma, Bucureşti: Editura Expert

Ionescu, M., Stănescu, S., M. (2014). Politici publice pentru romi. Raport de evaluare a programelor naționale finanțate de Uniunea Europeană pentru incluæịnea romilor, București: Editura Pro Universitaria

UNDP (2015). Decade of Roma inclusion: Progress Report

The World Bank (2014). Acbieving Roma inclusion in Romania: What does it take?

Zamfir, C, Preda M (coord) (2002). Romii în România, Bucureşti: Editura Expert

Zamfir, E., Zamfir, C. (coord.) (1993) Ţiganii intre ignorare și ingrijorare, Bucureşti: Editura Alternative.

Zamfir, E., Stănescu, S. M., Arpinte, D. (coord.) (2015). Asistența socială în România după 25 de ani: răspuns la problemele tranzititiei - texte selectate. Cluj-Napoca: Editura Școala Ardeleană 


\begin{tabular}{|c|c|c|c|c|}
\hline \multicolumn{5}{|c|}{ Table A 1. Appendix Roma Projects in Romania } \\
\hline \multirow[t]{4}{*}{ International } & $\begin{array}{l}\text { FRA } \text { Survey } \\
\text { (BG, CZ, HU, } \\
\text { RO, SK, FR, } \\
\text { GR, IT, PL, } \\
\text { PT, ES) }\end{array}$ & $\begin{array}{l}2011, \\
2008\end{array}$ & $\begin{array}{l}\text { Roma population } \\
1100 \text { and about } 500 \\
\text { Non-Roma }\end{array}$ & $\begin{array}{l}\text { education, employment, health, } \\
\text { dwelling, poverty, discrimination } \\
\text { and rights awareness. }\end{array}$ \\
\hline & $\begin{array}{l}\text { The Regional } \\
\text { Roma } \\
\text { UNDP/World } \\
\text { Bank/EC } \\
\text { Regional } \\
\text { Roma Survey } \\
\text { (BG, CZ, HU, } \\
\text { RO, SK, HR, } \\
\text { FYROM, MD, } \\
\text { AL, CS, BH) }\end{array}$ & 2011 & $\begin{array}{l}\text { About } 750 \text { Roma } \\
\text { and } 350 \text { Non-Roma } \\
\text { households by } \\
\text { country }\end{array}$ & $\begin{array}{l}\text { Household members profile, early } \\
\text { childhood education and care, status } \\
\text { of the household (dwelling type, } \\
\text { access to basic infrastructures, } \\
\text { household items possession etc.), } \\
\text { individual status and attitudes, } \\
\text { management section (interviewer's } \\
\text { evaluation of settlement characteristics } \\
\text { and housing conditions; identification } \\
\text { of the respondent; assessment of the } \\
\text { interview), incomes and expenditures } \\
\text { present both in the individual and } \\
\text { household module }\end{array}$ \\
\hline & $\begin{array}{l}\text { UNDP } \\
\text { Vulnerable } \\
\text { Groups } \\
\text { Survey } \\
\text { (AL, BH, BG, } \\
\text { HR, CS, RO) }\end{array}$ & 2004 & $\begin{array}{l}700 \text { households } \\
\text { (Roma, refugees, } \\
\text { IDPs and majority) }\end{array}$ & $\mathrm{NA}$ \\
\hline & $\begin{array}{l}\text { UNDP and } \\
\text { ILO Survey } \\
(\mathrm{BG}, \mathrm{CZ}, \mathrm{HU} \text {, } \\
\text { RO, SK) }\end{array}$ & 2001 & & NA \\
\hline \multirow[t]{2}{*}{ National } & $\begin{array}{l}\text { Open Society } \\
\text { Foundation, } \\
\text { Roma from } \\
\text { Romania, } \\
\text { Bulgaria, Italy } \\
\text { and Spain - } \\
\text { Between social } \\
\text { inclusion and } \\
\text { migration } \\
\text { Survey }\end{array}$ & 2012 & $\begin{array}{l}\text { Roma self-identified } \\
\text { of various } \\
\text { citizenships living in } \\
\text { Romania, Bulgaria, } \\
\text { Spain and Italy }\end{array}$ & $\begin{array}{l}\text { Profile of the respondent, activity, } \\
\text { employed persons, unemployed, } \\
\text { discrimination, social inclusion, } \\
\text { income, migration experience, } \\
\text { migration intentions, ethnic affiliation }\end{array}$ \\
\hline & $\begin{array}{l}\text { Open Society } \\
\text { Foundation, } \\
\text { The Situation } \\
\text { of Roma in } \\
\text { Romania } \\
\text { 2011. Between } \\
\text { social } \\
\text { inclusion and } \\
\text { migration }\end{array}$ & 2012 & $\begin{array}{l}\text { Roma population } \\
\text { and } 5 \text { case studies of } \\
\text { Roma subjects }\end{array}$ & $\begin{array}{l}\text { Community type, area, self- } \\
\text { identification of ethnic affiliation, } \\
\text { background characteristics, activity, } \\
\text { employed persons, type of occupation } \\
\text { and employment, unemployed, } \\
\text { discrimination, housing, migration } \\
\text { experience, migration intentions, } \\
\text { ethnic affiliation, income, household } \\
\text { composition }\end{array}$ \\
\hline
\end{tabular}




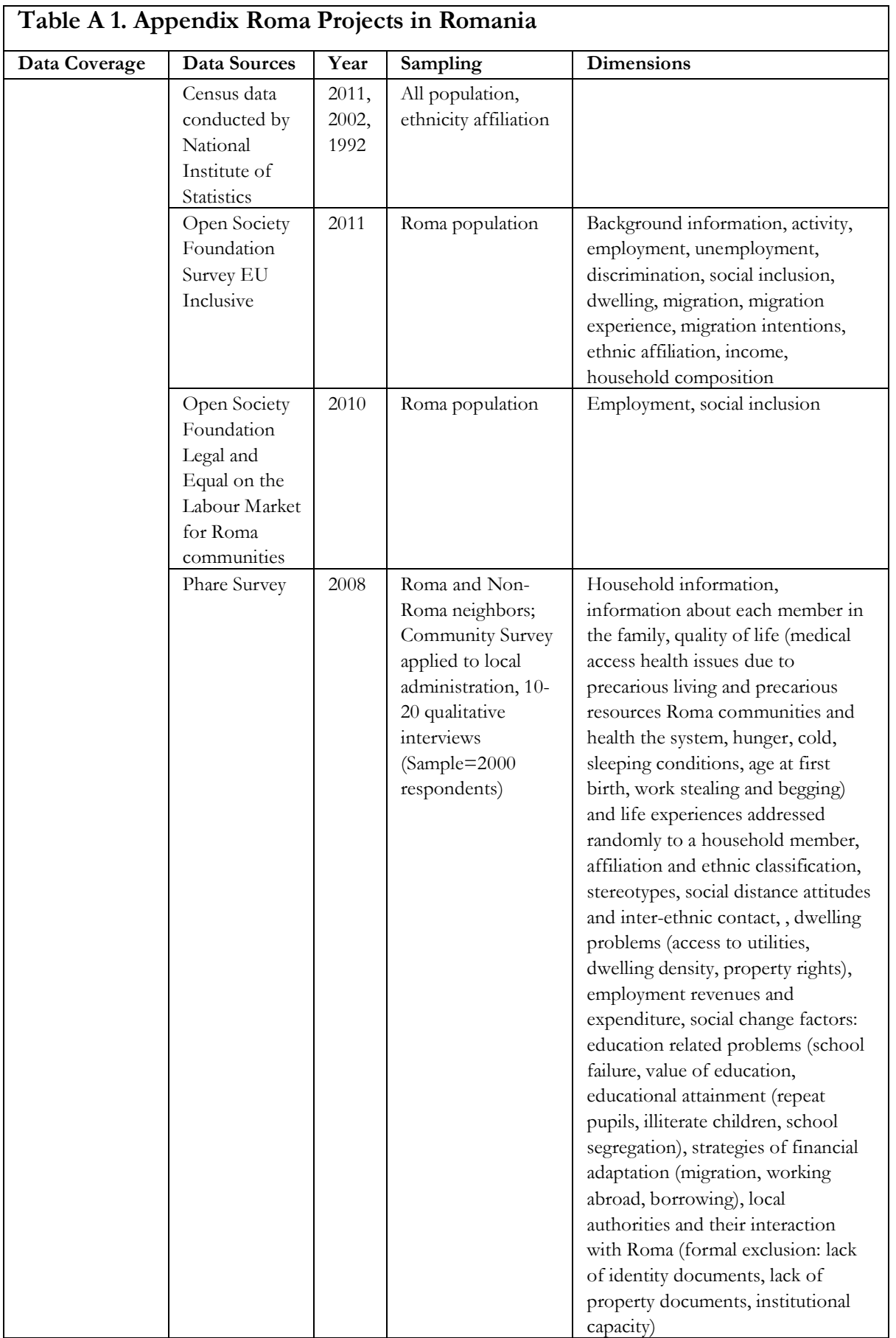




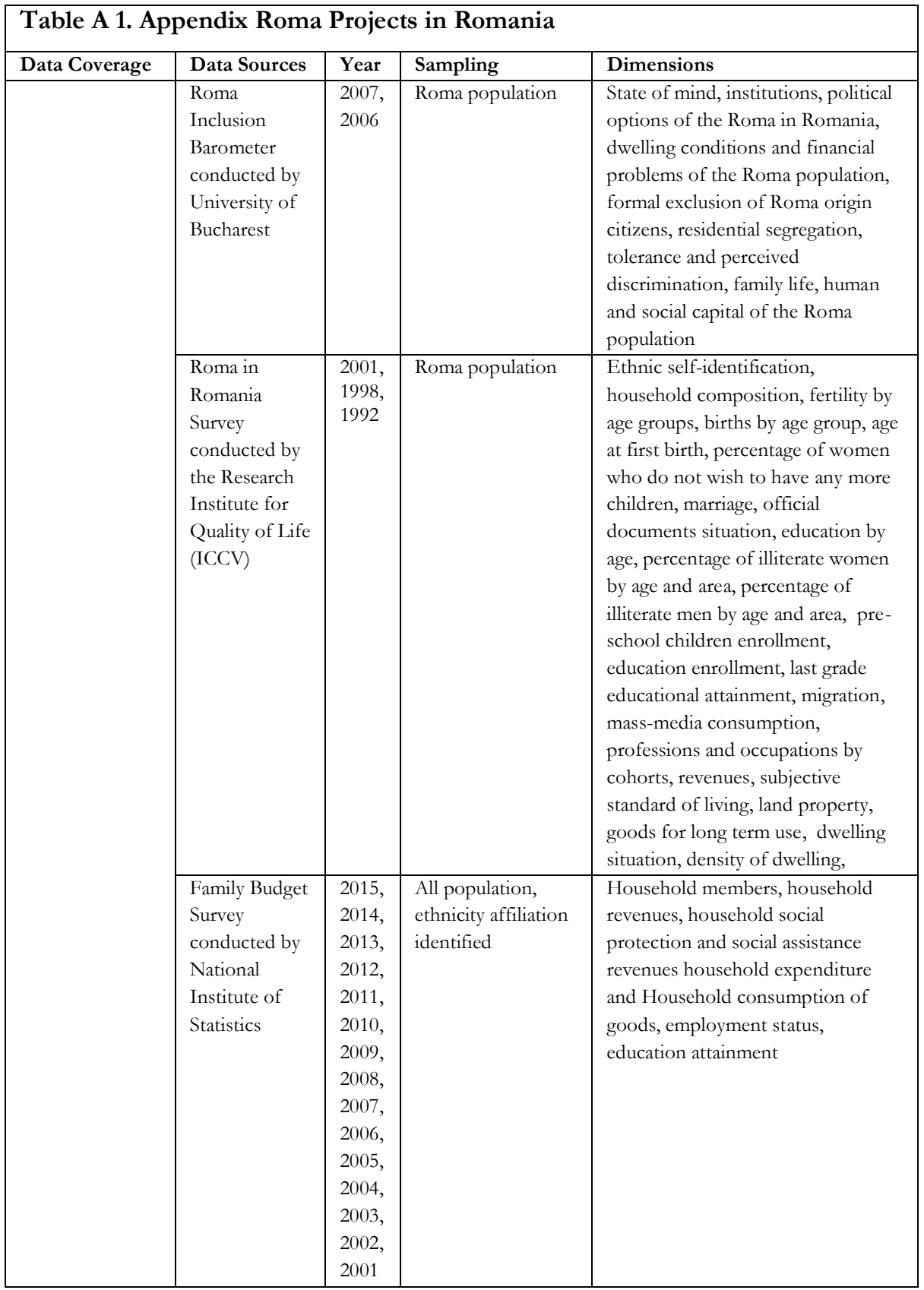


Dimensions of multiple deprivation in Roma population | 41

\begin{tabular}{|c|c|c|c|c|}
\hline \multirow[t]{3}{*}{ Data Coverage } & Data Sources & Year & Sampling & Dimensions \\
\hline & $\begin{array}{l}\text { ARACIP, } \\
\text { Education } \\
\text { Indicators, } \\
\text { RAQAPE } \\
2012 \text { the } \\
\text { National Map } \\
\text { of Educational } \\
\text { Risk }\end{array}$ & & School Data & $\begin{array}{l}\text { No of Roma children, Registry of } \\
\text { Formal Specialists in the field of } \\
\text { Education, }\end{array}$ \\
\hline & $\begin{array}{l}\text { Health } \\
\text { Indicators }\end{array}$ & & & \\
\hline \multirow[t]{2}{*}{ Regional/Local } & $\begin{array}{l}\text { UNICEF } \\
\text { Aurora Survey }\end{array}$ & 2015 & $\begin{array}{l}50.000 \text { Roma } \\
\text { households in } 45 \\
\text { communities }\end{array}$ & $\begin{array}{l}\text { Household composition and } \\
\text { demographics (including highest } \\
\text { attained educational level and } \\
\text { ethnicity), human capital investment } \\
\text { through non-formal activities with } \\
\text { the parent, children taken care of by } \\
\text { maternal assistant or } \\
\text { institutionalized, children } \\
\text { reintegrated after exiting the social } \\
\text { protection system, revenue sources, } \\
\text { health, hygiene, nutrition and risky } \\
\text { behavior, children education, } \\
\text {,monitoring of children health } \\
\text { status by regular general practitioner } \\
\text { check-ups and controls, nutrition, } \\
\text { health, nutrition and sex education } \\
\text { of teenagers, contraception and } \\
\text { births of all women in the } \\
\text { household over the age of 10, work } \\
\text { sharing practices in households, } \\
\text { teenage mothers, household level } \\
\text { data on social benefits, revenues } \\
\text { and expenditure, remittances, } \\
\text { dwelling conditions, parental } \\
\text { practices, dwelling conditions and } \\
\text { hygiene level of the household } \\
\text { assessed by the social worker, } \\
\text { vulnerabilities (poverty, health, } \\
\text { education, risky behavior, dwelling, } \\
\text { family and house social conditions) }\end{array}$ \\
\hline & $\begin{array}{l}\text { OSF, ISPMN } \\
\text { and CCRIT } \\
\text { SocioRoMap } \\
\text { Study }\end{array}$ & $\begin{array}{l}2014- \\
2016\end{array}$ & $\begin{array}{l}\text { Municipality } \\
\text { questionnaire, } \\
\text { Roma } \\
\text { Community/Group } \\
\text { questionnaire, } \\
\text { Health Mediator } \\
\text { questionnaire, } \\
\text { NGO-s } \\
\text { questionnaire }\end{array}$ & \\
\hline
\end{tabular}


Table A 1. Appendix Roma Projects in Romania

\begin{tabular}{|l|l|l|l|l|}
\hline Data Coverage & Data Sources & Year & Sampling & Dimensions \\
\hline & $\begin{array}{l}\text { World Bank } \\
\text { and University } \\
\text { of Bucharest } \\
\text { The Roma }\end{array}$ & 2005 & $\begin{array}{l}\text { Roma community } \\
\text { survey }\end{array}$ & $\begin{array}{l}\text { Living conditions, infrastructure, } \\
\text { income, dwelling infra-structure, } \\
\text { education, migration and poverty, } \\
\text { main income source by community } \\
\text { type, hierarchy of Roma problems } \\
\text { as perceived by local experts, } \\
\text { poverty factors and their linkage } \\
\text { Social Map } \\
\text { Survey, } \\
\text { PROROMA }\end{array}$ \\
\end{tabular}

